\title{
Avaliação de resultados tardios com bioprótese de aorta heteróloga porcina
}

Renato A. K. KALIL*, João Ricardo SANT'ANNA*, Júlio E. SCHOER ${ }^{\star \star}$, Paulo Roberto PRATES ${ }^{\star \star}$, Fernando A. LUCCHESE**, Edemar M. PEREIRA**, Altamiro R. COSTA**, Ivo A. NESRALLA*

RBCCV 44205-80

KALIL, R. A. K.; SANT'ANNA, J. R.; SCHOER, J. E.; PRATES, P. R.; LUCCHESE, F. A.; PEREIRA, E. M.; COSTA, A. R.; NESRALLA, I. A. - Avaliaçăo de resultados tardios com bioprótese de aorta heteróloga porcina. Rev. Bras. Cir. Cardiovasc., 4(1): 84-89, 1989.

RESUMO: Foi analisada a evolução de 150 pacientes operados entre 1982 e 1988, com bioprótese porcina. Eram 62 do sexo feminino e 88 do masculino, idade média de 51,6 (15 a 81) anos. Nove estavam em classe funcional II, 120 em III e 21 em IV; 46 mitrais, 50 ab́rticos, 80 múltiplos e 24 associados a revascularização do miocárdio (5 mitrais e 19 aórticos). A mortalidade hospitalar foi de $12 \%$ (18 casos). Vinte e sete não foram acompanhados no pós-operatório tardio. A mortalidade tardia foi de 2,6\% (4 casos), por insuficiência renal, meningoencefalite, insuficiência cardiaca congestiva e embolia pulmonar. Foram reoperados $5(3,3 \%)$, por: endocardite infecciosa, pertuitos paravalvulares e falência primária do tecido valvular em 2 casos, cujas idades eram 15 e 25 anos. Três pacientes apresentaram sinais de regurgitaçăo leve, mas não foi indicada reintervençăo. As curvas atuariais, até o sétimo ano de pós-operatório, mostraram probabilidade de sobrevida de $82,9 \pm 3,7$ anos e livres de reoperação, $70,1 \pm 6,7 \%$. Para aórticos isolados, foi: $88,0 \pm 5,4 \%$ e $60,4 \pm 17,4 \%$, respectivamente. Para os mitrais: $87,3 \pm 5,6 \%$ e $70,9 \pm 11,1 \%$. Atualmente, há, entre 101 casos, 82 em classe I, 17 em II e 2 em III. Falhas das biopróteses ocorreram em $21,4 \%$ dos pacientes com menos de 30 anos, $1,1 \%$ entre 31 e 60 anos e $2 \%$ naqueles com mais de 60 anos. Foram mais freqüentes falhas no sexo masculino $(4,5 \%)$ que no feminino $(1,6 \%)$. Não houve diferença nas posiçōes mitral ou aórtica. A bioprótese estudada apresentou bons resultados clínicos no período de evoluçăo até 7 anos. Sua durabilidade é comparável às demais biopróteses, sendo as falhas, na maioria, devidas a causas conhecidas, em baixa incidência. Estudos a mais longo prazo serão úteis para definir a tendência de evolução futura.

DESCRITORES: próteses valvulares cardiacas, biológicas; próteses valvulares cardiacas, cirurgia.

\section{INTRODUÇÃO}

Apesar dos contínuos avanços na tecnologia de construçāo de substitutos valvulares cardíacos, ainda não dispomos do dispositivo ideal. As próteses metálicas oferecem vantagens hemodinâmicas e de durabilidade, enquanto as biológicas têm o apelo da não-trombogenicidade, porém seriam de duraçăo limitada. No balanço das complicações, entretanto, parece haver equilibrio quanto ao resultado final, em termos de sobrevida. Este fato, aliado à melhor qualidade de vida experimentada pelos portadores de biopróteses, tem favorecido seu emprego, em muitos Serviços, sempre que as condiçōes do paciente permitem, principalmente em relaçāo à sua idade e ao tamanho da prótese a implantar.

Enxertos valvulares autógenos e homólogos têm sido pouco utilizados, por dificuldades de obtenção e armazenamento. As biopróteses mais implantadas hoje são a valva aórtica de porco ${ }^{1,3}$ e a válvula confeccionada

\footnotetext{
Trabalho realizado no Instituto de Cardiologia do Rio Grande do Sul. Fundą̧ăo Universitária de Cardiologia. Porto Alegre, RS, Brasil. Recebido para publicaçắo em 10 de abril de 1989.

- Do Instituto de Cardiologia do Rio Grande do Sul. Fundação Universitária de Cardiologia e da Universidade Federal do Rio Grande do Sul.

** Do Instituto de Cardiologia do Rio Grande do Sul. Fundação Universitária de Cardiologia.

Endereço para separatas: Renato A. K. Kalil. Av. Princesa Isabel, 395. 90620 Porto Alegre, RS, Brasil.
} 
KALIL, R. A. K.; SANT'ANNA, J. R.; SCHOER, J. E.; PRATES, P. R.; LUCCHESE, F. A.; PEREIRA, E. M.; COSTA, A. R.; NESRALLA, I. A. - Avaliação de resultados tardios com bioprótese de aorta heteróloga porcina. Rev. Bras. Cir. Cardiovasc., 4(1): 84-89, 1989.

com pericárdio bovino ${ }^{4}$, ambas preservadas em glutaraldeído e montadas em suportes flexíveis. Há modelos de várias procedências disponiveis, nacionais e estrangeiras. Neste trabalho, procuramos avaliar os resultados obtidos no Instituto de Cardiologia do Rio Grande do Sul, com um modelo de valva aórtica de porco preservada em glutaraldeído, de produção por laboratório nacional, visando obter parâmetros de comparação com outros modelos, para o objetivo final de melhor embasar a escolha, pelo cirurgião, do substituto a ser implantado.

\section{CASUÍSTICA E MÉTODOS}

No período de 1982 a 1988, 150 pacientes foram submetidos a substituição valvar por bioprótese de aorta heteróloga preservada em glutaraldeído*, nas posiçōes aórtica e/ou mitral. A idade média foi de 51,6 (15 a 81) anos, sendo 62 do sexo feminino e 88 do masculino. Nove pacientes estavam em classe funcional II, por ocasião da cirurgia, $120 \mathrm{em}$ classe III e 21 em classe IV. $\mathrm{Na}$ posição mitral, isoladamente, foram feitos 46 implantes; na aórtica, 50 implantes; em mais de uma posição, 30 implantes, além de 24 casos com ponte de safena associada, dos quais 5 eram mitrais e 19 aórticos.

Os pacientes foram operados com auxílio de circulação extracorpórea e hipotermia sistêmica leve a moderada $\left(28-32^{\circ} \mathrm{C}\right)$, utilizando-se oxigenadores de bolhas descartáveis e parada isquêmica do coração sob proteção com solução cardioplégica infundida na aorta ascendente, ou nos óstios coronarianos, conforme o caso, associada a hipotermia tópica com solução salina.

Os resultados foram analisados no período de janeiro a março de 1989, através de revisão dos prontuários daqueles pacientes acompanhados no próprio hospital, ou por contato direto com os médicos-assistentes, ou, ainda, por correspondência enviada aos pacientes, com o objetivo de verificar a mortalidade tardia, a incidência de reoperaçōes e suas causas, além da classe funcional no pós-operatório tardio. A mortalidade imediata foi computada até o trigésimo dia de pós-operatório. Foi analisado o grupo de forma global, além de, separadamente, aqueles em que foi realizada substituição valvar isolada, sem procedimentos associados. Os dados de sobrevida total e sem reoperações foram submetidos a análise atuarial e expressos em gráficos próprios, representando-se a média \pm erro-padrão. Entre os pacientes submetidos a reoperaçōes, ou que foram a óbito, foram analisados, comparativamente: idade na época do implante, sexo, posição da válvula, tempo de acompanhamento e causa da falha do tratamento.

\footnotetext{
- Laboratório Biocor, Belo Horizonte, MG, Brasil.
}

\section{RESULTADOS}

Dos 150 pacientes operados, 18 evoluíram para óbito, nos primeiros 30 dias, totalizando a mortalidade imediata global em $12 \%$. Dos restantes 132 pacientes, 27 foram perdidos para acompanhamento e 105 tiveram sua evolução acompanhada por períodos mínimos de 1 ano e máximos de 7 anos. Ocorreram 4 óbitos tardios $(2,6 \%)$, devidos a insuficiência renal, meningoencefalite, insuficiência cardíaca congestiva e embolia pulmonar. Em 1 paciente, não foi diagnosticada a causa mortis (Tabela 1).

Foram necessárias reoperaçōes para substituição valvular em $5(3,3 \%)$ pacientes (Tabela 2$)$, por diversas causas: 1 por endocardite infecciosa aos 4 meses de pós-operatório, 2 por deiscência paravalvular aos 1 a 2 meses e aos 3 a 5 meses de pós-operatório (nos quais o tecido da bioprótese não estava alterado) e 2 por falência primária do tecido. Estes 2 pacientes que desenvolveram falência primária do tecido foram operados em idades de 15 a 25 anos, respectivamente. Outros 3 pacientes apresentam sinais de regurgitação leve a moderada, mas não tiveram indicada reoperação até o encerramento deste trabalho; estão todos no $6^{\circ}$ ano de acompanhamento e suas idades, no momento da cirurgia, eram 18,48 e 64 anos, respectivamente, com biopróteses mitro-aórtica, mitral a aórtica em cada caso (Tabela 3).

A probabilidade de sobrevida no grupo total, avaliada por análise atuarial, foi de $82,9 \pm 3,5 \%$ aos 7 anos, sendo de $70,1 \pm 6,7 \%$ a probabilidade de sobrevida sem reoperação para substituição valvular (Figura 1). Para o grupo de substituição aórtica isolada, a probabilidade de sobrevida aos 7 anos foi de $88,0 \pm 5,4 \%$. Livres de reoperação, nesse grupo, prevê-se 60,4 \pm $17,4 \%$ no período (Figura 2). No grupo de substituição mitral isolada, as probabilidades são de $87,3 \pm 5,6 \%$ para sobrevida e de $70,9 \pm 11,1 \%$ livres de reoperação (Figura 3). Deve ser salientado o erro-padrão maior nas estimativas após o quinto ano de pós-operatório, devido ao menor número de Dacientes nesses períodos.

$\mathrm{Na}$ análise da situação clínica atual dos 101 pacientes acompanhados, encontramos $82 \mathrm{em}$ classe funcional I, 17 em classe funcional II e 2 em classe III (Figura 4).

Em 2 pacientes reoperados, foi constatada rotura do tecido por falência primária ou degeneração. Em 3 outros, ainda não operados, há regurgitação valvular, muito provavelmente devida à mesma causa, embora não tenhamos comprovação. Nos demais pacientes operados, não havia sinais degenerativos nas biopróteses. Por outro lado, as causas de b́bito tardio não parecem ter sido devidas a falhas das biopróteses. Dessa forma, para analisar prováveis fatores causais de falência primária dos tecidos, temos 5 pacientes (Tabela 4), nos 
KALIL, R. A. K.; SANT'ANNA, J. R.; SCHOER, J. E.; PRATES, P. R.; LUCCHESE, F. A.; PEREIRA, E. M.; COSTA, A. R.; NESRALLA, I. A. - Avaliação de resultados tardios com bioprótese de aorta heteróloga porcina. Rev. Bras. Cir. Cardiovasc., 4(1): 84-89, 1989.

TABELA 1

FATORES RELACIONADOS A MORTALIDADE TARDIA

\begin{tabular}{|c|c|c|c|c|c|c|c|c|}
\hline Casos $\mathrm{N}^{\circ}$ & Idade (Anos) & Sexo & Data Cirurgia & Posição & Etiologia & Data Obito & Causa de Óbito & Tempo p.o. \\
\hline 16 & 20 & $M$ & $30-06-82$ & aórtica & $\begin{array}{l}\text { Endocardite } \\
\text { infecciosa }\end{array}$ & $04-01-84$ & $\begin{array}{l}\text { Insuficiência renal e } \\
\text { meningoencefalite }\end{array}$ & $18 \mathrm{~m}$ \\
\hline 22 & 56 & M & $30-11-82$ & mitral & Reumática & $10-01-84$ & $\begin{array}{c}\text { Insuficiência cardiaca } \\
\text { congestiva }\end{array}$ & $14 \mathrm{~m}$ \\
\hline 103 & 71 & $M$ & $04-06-86$ & aórtica & Escleros senil & $04-12-86$ & Embolia pulmonar & $4 \mathrm{~m}$ \\
\hline 146 & 45 & $\mathrm{~F}$ & $04-03-88$ & mitral & Reumática & $14-03-89$ & Desconhecida & $12 \mathrm{~m}$ \\
\hline
\end{tabular}

TABELA 2

FATORES RELACIONADOS A REOPERAÇŌES

\begin{tabular}{|c|c|c|c|c|c|c|c|}
\hline Casos $\mathrm{N}^{\circ}$ & Idade (Anos) & Sexo & Data Cirurgia & Data Reoperaçāo & Posição & Causa & Tempo p.o. \\
\hline 101 & 53 & $F$ & $23-06-86$ & $25-08-87$ & Mitral & $\begin{array}{l}\text { Deiscência paravalvular } \\
\text { Prótese sem alteraçāo }\end{array}$ & $14 \mathrm{~m}$ \\
\hline 07 & 25 & M & $19-04-82$ & $23-06-86$ & Aórtica & Rotura 1 folheto & 4 a $4 m$ \\
\hline 12 & 15 & M & $16-04-82$ & $17-06-86$ & Mitral & $\begin{array}{c}\text { Degeneração e laceração } \\
\text { dos folhetos }\end{array}$ & 4 a $2 m$ \\
\hline 38 & 54 & M & $23-08-84$ & $09-12-87$ & Aórtica & $\begin{array}{l}\text { Deiscência paravalvular } \\
\text { Prótese sem alteraçāo }\end{array}$ & 3 a $5 \mathrm{~m}$ \\
\hline 131 & 26 & $F$ & $31-03-87$ & $10-07-87$ & Mitral & Endocardite infecciosa & $4 m$ \\
\hline
\end{tabular}

TABELA 3

CASOS DE DISFUNÇĀO AO DIAGNÓSTICO CLÍNICO, NĀO REOPERADOS

\begin{tabular}{rccccc}
\hline $\begin{array}{c}\text { Casos } \\
\text { No }\end{array}$ & $\begin{array}{c}\text { Idade } \\
\text { Anos }\end{array}$ & Sexo & Posiçāo & $\begin{array}{c}\text { Data } \\
\text { Cirurgia }\end{array}$ & $\begin{array}{c}\text { Tempo } \\
\text { p.o. }\end{array}$ \\
\hline 8 & 48 & F & Mitral & abril 82 & 5 a $9 \mathrm{~m}$ \\
17 & 18 & M & Mitral e Aórtica & julho 82 & 5 a $4 \mathrm{~m}$ \\
18 & 64 & M & Aórtica & agosto 82 & 5 a $5 \mathrm{~m}$ \\
\hline
\end{tabular}

TABELA 4

DISFUNÇĀO POR FALENNCIA PRIMARIA DO TECIDO POR GRUPOS ETARIOS

\begin{tabular}{cccr}
\hline Idade & Número de Casos & Número Disfunçâo & $\%$ \\
\hline$>30$ anos & 14 & 3 & $21,4 \%$ \\
31 a 60 anos & 87 & 1 & $1,1 \%$ \\
$>61$ anos & 49 & 1 & $2,0 \%$ \\
\hline
\end{tabular}

quais a idade, por ocasião do implante, parece ter sido fator importante. Em idades inferiores a 30 anos, ocorreram $21,4 \%$ de falhas, enquanto nas faixas etárias superiores o indice foi de 1 a $2 \%$.

Quanto ao sexo, ocorreram 4 (4,5\%) falências em pacientes do sexo masculino e 1 apenas $(1,6 \%)$ no sexo feminino. Quanto à posição da bioprótese implantada, ocorreram $2(3,9 \%)$ falências, no total de 51 mitrais (incluindo os casos de revascularização miocárdica). Para a posição aórtica, ocorreram $2(2,9 \%)$ falências em 69 implantes, um $(3,3 \%)$ caso de falência de tecido ocorreu no grupo de implantes múltiplos, composto de $30 \mathrm{pa}$ cientes.

\section{DISCUSSÃO}

A era dos modernos substitutos valvulares biológicos heterólogos teve início em 1965, quando do pri-

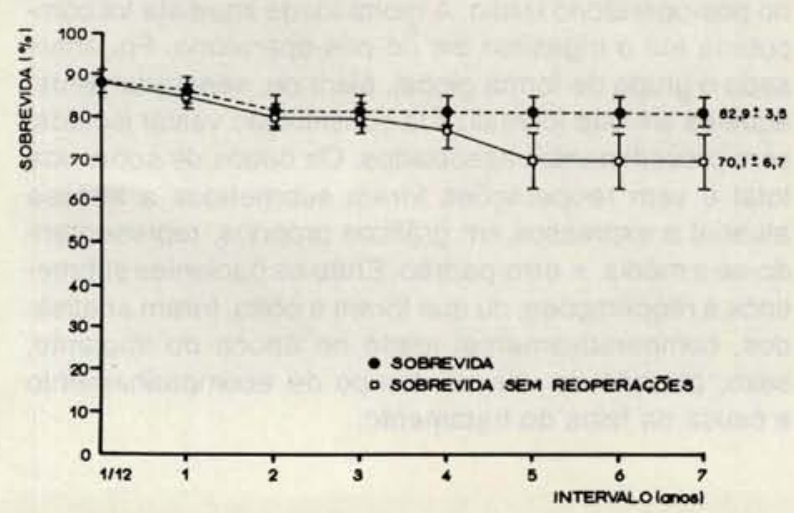

Fig. 1 - Probabilidade de sobrevida total e sobrevida sem reoperaçōes, por análise atuarial de todos os pacientes estudados. 

I. A. - Avaliação de resultados tardios com bioprótese de aorta heteróloga porcina. Rev. Bras. Cir. Cardiovasc., 4(1): 84-89, 1989.

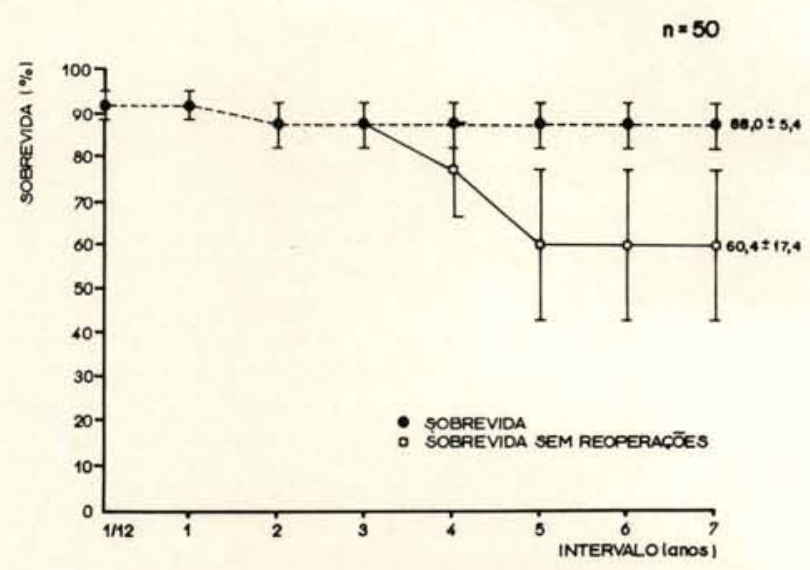

Fig. 2 - Probabilidade de sobrevida, total e sem reoperaçăo, por análise atuarial no grupo de pacientes submetidos a substituiçăo isolada da valva aórtica.

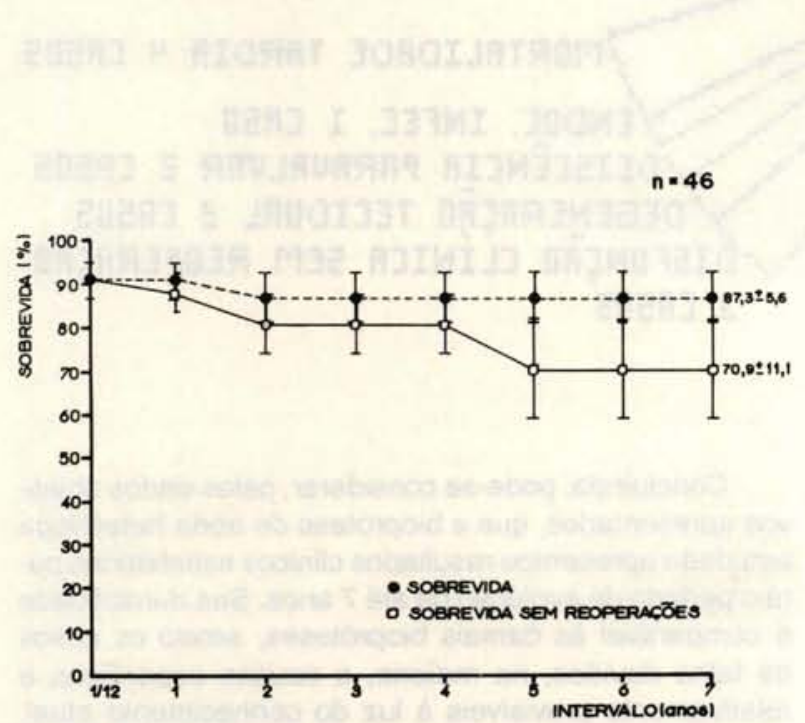

Fig. 3 - Probabilidade de sobrevida, total e sem reoperaçóes, por análise atuarial, no grupo de pacientes submetidos a substituiçăo mitral isolada.

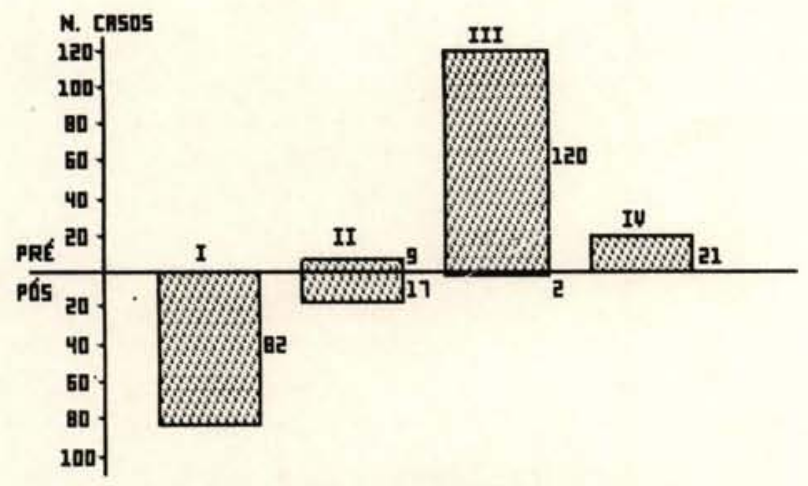

Fig. 4-Classe funcional pré e pós-operatória. meiro implante aórtico de um enxerto heterólogo ${ }^{2}$. Os métodos de preservaçāo, montagem a armazenamento têm sido continuamente aperfeiçoados, tendo, no entanto, sua base na experiência inicial de CARPENTIER et alii ${ }^{3}$.

As biopróteses atuais, assim chamadas por perderem as características de simples enxertos e se aproximarem mais de uma prótese artificial, conservam em comum a montagem em suportes flexiveis e a preservação em glutaraldeído. Diversos laboratórios nacionais e estrangeiros comercializam biopróteses de aorta heteróloga, cujos resultados clínicos imediatos costumam manter uniformidade, e as experiências a longo prazo têm sido objeto de reavaliaçōes recentes ${ }^{1,6,7}$.

No Instituto de Cardiologia do Rio Grande do Sul, além das próteses mecânicas, já foram empregadas válvulas de dura-máter, de pericárdio bovino e de aorta porcina preservadas em glutaraldeído, de variadas procedências. Neste trabalho, é analisada uma dessas biopróteses de produção nacional, com o objetivo, já expresso, de se obterem dados para a escolha mais consciente e objetiva, pelo cirurgião, do substituto a utilizar.

A mortalidade imediata de $12 \%$, nesta série, não esteve relacionada com o modelo da bioprótese, situando-se na média aceitável para o grupo de cardiopatias orovalvares, nāo selecionado, aqui analisado. Os óbitos tardios, na incidência de $2,6 \%$, da mesma forma, não ocorreram por disfunção valvular, pelo menos nos casos de evolução e causa mortis conhecidas.

Disfunçōes valvulares ocorreram por causas variadas. Os casos de endocardite infecciosa e de deiscência paravalvular não são atribuiveis diretamente ao modelo da bioprótese. Ocorreram, nesta série, em número relativamente pequeno, mas foram responsáveis por reoperaçōes. Merecem análise mais detalhada de casos de falência primária do tecido. Neste grupo, se incluírmos os 2 casos de reoperação somados aos 3 de regurgitaçāo valvular não reoperados, teremos 5 pacientes em 105 com acompanhamento atualizado (cerca de $5 \%$ de falência primária de tecido). Deve-se considerar que, nos pacientes não reoperados, não temos a comprovação morfológica da falha tecidual, porém, para análise criteriosa, devem ser computados como tal. Como dado geral, essa incidência de falha tecidual é baixa para o período de evolução estudado. No início da experiência e mesmo recentemente, muitas vezes fomos forçados a implantar biopróteses em pacientes jovens, por situaçōes clínicas, pessoais, ou sociais especificas. Sabe-se que a degeneração do tecido biológico é mais precoce e mais freqüente em jovens ${ }^{5}$. Dos 5 pacientes citados, 3 tinham menos de 30 anos na época do implante. Em toda a experiência, a diferença entre faixas etárias acima ou abaixo dos 30 anos, para disfunção valvular foi altamente significativa. Portanto, podemos concluir que a idade desempenhou papel importante no aumento dos casos de falha tecidual, a exemplo do que outros autores já relataram para biopróteses semelhantes ${ }^{5,6}$. 


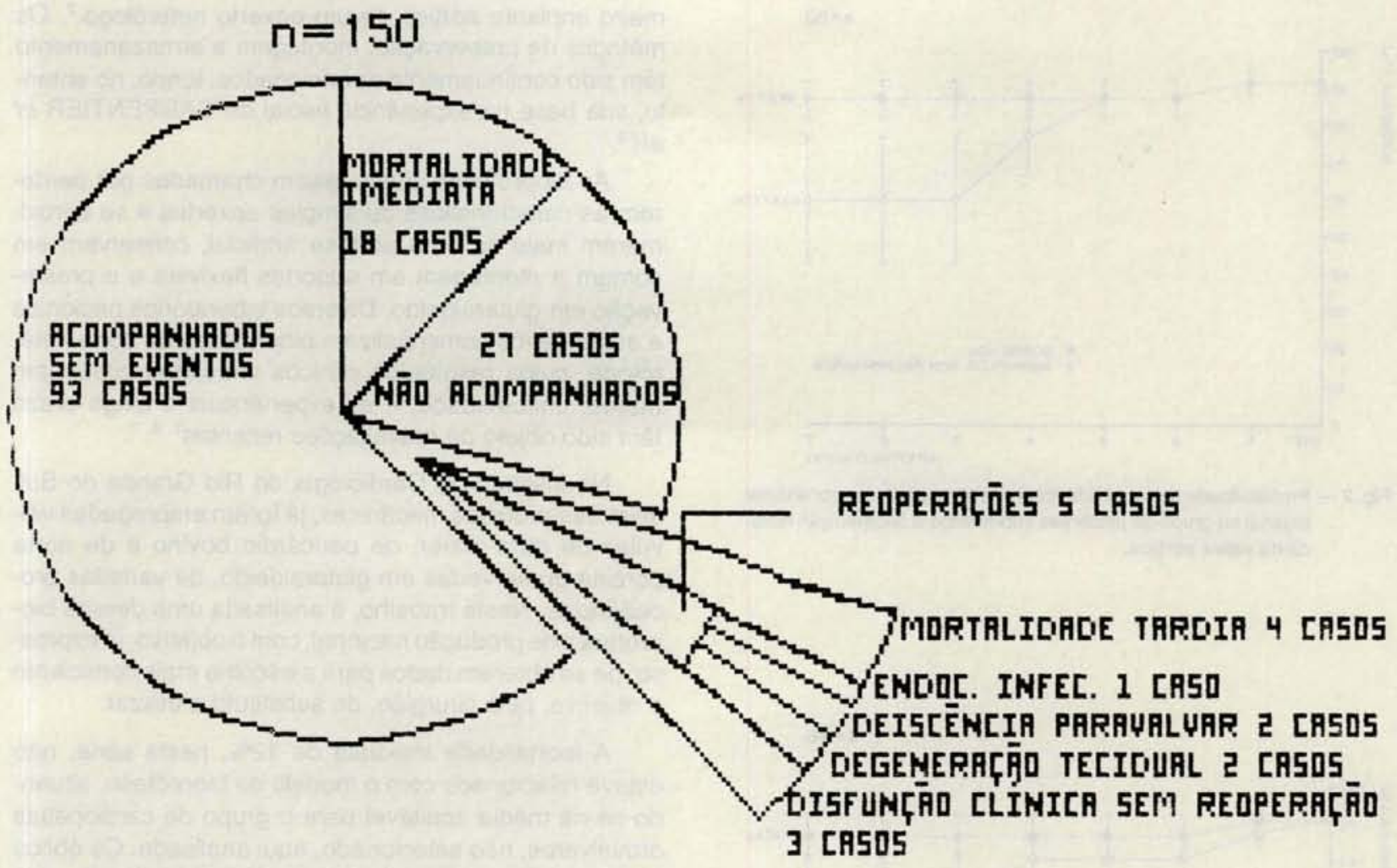

Fig. 5 - Representação esquemática da experiência global

As probabilidades de sobrevida total e de sobrevida sem reoperaçōes encontradas nas curvas atuariais foram satisfatórios para o período estudado, nos diversos grupos. Entre os aórticos, a sobrevida sem reoperaçōes foi menos favorável. Entretanto, quanto à durabilidade tecidual, apenas 1 dos casos de falha ocorreu por rotura de tecido da bioprótese na posição aórtica. $\mathrm{Na}$ análise do índice de falências, não houve diferença significativa entre as posiçōes mitral ou aórtica. Por motivos não esclarecidos, a ocorrência de falhas foi maior no sexo masculino, em relaçăo ao feminino.
Concluindo, pode-se considerar, pelos dados objetivos apresentados, que a bioprótese de aorta heteróloga estudada apresentou resultados clínicos satisfatórios para o período de evolução de até 7 anos. Sua durabilidade é comparável às demais biopróteses, sendo os casos de falha devidos, na maioria, a causas específicas e relativamente previsíveis à luz do conhecimento atual. Estudos a mais longo prazo serão necessários, para avaliar a tendência de evolução futura. 
KALIL, R. A. K.; SANT'ANNA, J. R.; SCHOER, J. E.; PRATES, P. R.; LUCCHESE, F. A.; PEREIRA, E. M.; COSTA, A. R.; NESRALLA, I. A. - Avaliação de resultados tardios com bioprótese de aorta heteróloga porcina. Rev. Bras. Cir. Cardiovasc., 4(1): 84-89, 1989.

RBCCV 44205-80

KALIL, R. A. K.; SANT'ANNA, J. R.; SCHOER, J. E.; PRATES, P. R.; LUCCHESE, F. A.; PEREIRA, E. M.; COSTA, A. R.; NESRALLA, I. A. - Late results with an heterologous aortic porcine bioprosthesis Rev. Bras. Cir. Cardiovasc., 4(1): 84-89, 1989.

ABSTRACT: The clinical results of an aortic heterograft model (Biocor) was evaluated in a series of 150 patients, operated upon from 1982 to 1988 and followed-up for until 7 years post-operatively. Sixty-two were female and 88 male. Age ranged from 15 to $81(m=51.6)$ years. There were 9 in functional class II, 120 in III and 21 in IV. In the isolated mitral position there were 46 implants, aortic isolated 50 , multiple 30 and 24 associated with myocardial revascularization (5 mitral and 19 aortic). Hospital mortality was $12 \%$ (18 cases). Twenty seven patients were lost for late follow-up. Late mortality was $2.6 \%$ ( 4 cases), caused by rena! failure, meningoencefalitis, congestive heart failure and pulmonary embolism. There were $5(3.3 \%)$, due to infectious endocarditis, paravalvular leaks, primary tissue failure (2 cases at ages 15 and 25). Three others patients presented clinical signs of mild regurgitation and were not reoperated. The actuarial survival curves showed a probability of survival and probability of no surgical events, respectively, for the whole group: $82.9 \pm 3.7$ years and $70.1 \pm 6.7$ years, for the aortic group: $88.0 \pm 5.6 \%$ and 60.4 $\pm 17.4 \%$, for the mitral group: $87.3 \pm 5.6 \%$ and $70.9 \pm 11.1 \%$. At the last clinical evaluation, there were 82 patients in functional class I, 17 in II and 2 in III. Bioprosthesis attributed failures occurred in $21.4 \%$ of the patients operated upon, younger than 30 years, $1.1 \%$ between 31 and 60 years, and $2 \%$ in the older than 61 years. Male sex was related to $4.5 \%$ of failures and female, $1.6 \%$. There was no difference between the mitral, aortic, or multiple groups. The bioprosthesis evaluated, for a follow-up of 7 years, presented good results. Causes of failure were usually related to age, infection or leaking, at a low incidence in the study period. Longer observation, however, is still advisable for definitive conclusions.

DESCRIPTORS: heart valves, biologic; heart valves prostheses, surgery.

\section{REFERÊNCIAS BIBLIOGRÁFICAS}

1 AMATO, M. C. M.; POMERANTZEFF, P.; GRINBERG, M.; BELLOTTI, G.; PILEGGI, F. - Evolução imediata e tardia de pacientes com bioprótese porcina. Arq. Bras. Cardiol., 51: 381-384, 1988.

2 BINET, J. P.; CARPENTIER, A.; LANGLOIS, J.; DURAN, C.; COLVEZ, P. - Implantation de valves hetérogènes dans le traitement de cardiopaties aortiques. C. $R$. Acad. Sc. Paris, 261: 5733-5734, 1965.

3 CARPENTIER, A.; LEMAIGRE, G.; ROBERT, L.; CARPENTIER, S.; DUBOST, C. - Biological factors affecting long-term results of valvular heterografts. J. Thorac. Cardiovasc. Surg., 58: 467-483, 1969.

4 IONESCU, M. I.; SMITH, D. R.; HASAN, S. S.; CHIDANBARAN, M.; TANDON, A. P. - Clinical durability of the pericardial xenograft valve: ten years experience with mitral replacement. An. Thorac. Surg., 34: 265-277, 1982.

5 JAMIESON, W. R. E.; ROSADO, L. J.; MUNRO, A. I.; GEREIN, A. N.; BURR, L. H.; MIYAGISHIMA, R. T.; JAMUSZ, M. T.; TYERS, G. F. O. - Carpentier-Edwards standard porcine bioprosthesis: primary tissue failure (structural valve deterioration) by age groups. An. Thorac. Surg.; 46: 155-162, 1988.

6 MAGILLIGAN, D. J.; LEWIS Jr., J. W.; STEIN, P.; ALAM, M. - The porcine bioprosthetic valve: experience at 15 years. Ann. Thorac. Surg., 48: 324-330, 1989.

7 MILANO, A. D.; BORTOLOTTI, V.; MAZZUCCO, A.; GUERRA, F.; STELLIN, G.; FALENTI, E.; THIENE, G.; GALUCCI, V. - Performance of the Hancock porcine bioprosthesis following aortic valve replacement: considerations based on a 15-year experience. Ann. Thorac. Surg., 46: 216-222, 1988. 